\title{
Pulmonary vascular resistance and compliance relationship in pulmonary hypertension
}

\author{
Denis Chemla ${ }^{1,2}$, Edmund M.T. Lau ${ }^{1,2,3}$, Yves Papelier ${ }^{2}$, Pierre Attal ${ }^{4}$ and \\ Philippe Hervé 5
}

\author{
Number 10 in the series "Physiology in respiratory medicine" \\ Edited by R. Naeije, D. Chemla, A. Vonk-Noordegraaf and A.T. Dinh-Xuan
}

\author{
Affiliations: \\ ${ }^{1}$ Univ. Paris-Sud, Faculté de Médecine, Inserm U_999, Le Kremlin Bicêtre, France. \\ ${ }^{2}$ AP-HP, Services des Explorations Fonctionnelles, Hôpital de Bicêtre, Le Kremlin Bicêtre, France. \\ ${ }^{3}$ Dept of Respiratory Medicine, Royal Prince Alfred Hospital, University of Sydney, Camperdown, Australia. \\ ${ }^{4}$ Dept of Otolaryngology-Head and Neck Surgery, Shaare-Zedek Medical Center and Hebrew University \\ Medical School, Jerusalem, Israel. \\ ${ }^{5}$ Centre Chirurgical Marie Lannelongue, Le Plessis-Robinson, France.
}

\section{Correspondence:}

Denis Chemla, Service des Explorations Fonctionnelles - Broca 7, Hôpital de Bicêtre, 78 rue du Général Leclerc, 94275 Le Kremlin Bicêtre, France.

E-mail: denis.chemlaabct.aphp.fr

ABSTRACT Right ventricular adaptation to the increased pulmonary arterial load is a key determinant of outcomes in pulmonary hypertension (PH). Pulmonary vascular resistance (PVR) and total arterial compliance (C) quantify resistive and elastic properties of pulmonary arteries that modulate the steady and pulsatile components of pulmonary arterial load, respectively. PVR is commonly calculated as transpulmonary pressure gradient over pulmonary flow and total arterial compliance as stroke volume over pulmonary arterial pulse pressure (SV/PApp). Assuming that there is an inverse, hyperbolic relationship between PVR and $\mathrm{C}$, recent studies have popularised the concept that their product (RC-time of the pulmonary circulation, in seconds) is "constant" in health and diseases. However, emerging evidence suggests that this concept should be challenged, with shortened RC-times documented in post-capillary $\mathrm{PH}$ and normotensive subjects. Furthermore, reported RC-times in the literature have consistently demonstrated significant scatter around the mean. In precapillary $\mathrm{PH}$, the true PVR can be overestimated if one uses the standard PVR equation because the zero-flow pressure may be significantly higher than pulmonary arterial wedge pressure. Furthermore, SV/PApp may also overestimate true C. Further studies are needed to clarify some of the inconsistencies of pulmonary RC-time, as this has major implications for our understanding of the arterial load in diseases of the pulmonary circulation.

@ERSpublications

Empiric estimates of pulmonary arterial load are prone to errors and may result in overestimation of RC-time http://ow.ly/QqtAV

\footnotetext{
Previous articles in this series: No. 1: Naeije R, Vachiery J-L, Yerly P, et al. The transpulmonary pressure gradient for the diagnosis of pulmonary vascular diseases. Eur Respir J 2013; 41: 217-223. No. 2: Hughes JMB, van der Lee I. The TL,NO/TL,CO ratio in pulmonary function test interpretation. Eur Respir J 2013; 41: 453-461. No. 3: Vonk-Noordegraaf A, Westerhof N. Describing right ventricular function. Eur Respir J 2013; 41: 1419-1423. No. 4: Hamzaoui O, Monnet X, Teboul J-L. Pulsus paradoxus. Eur Respir J 2013; 42: 1696-1705. No. 5: Prisk GK. Microgravity and the respiratory system. Eur Respir J 2014; 43: 1459-1471. No. 6: Dempsey JA, Smith CA. Pathophysiology of human ventilatory control. Eur Respir J 2014; 44: 495-512. No. 7: Petersson J, Glenny RW. Gas exchange and ventilation-perfusion relationships in the lung. Eur Respir J 2014; 44: 1023-1041. No. 8: Wagner PD. The physiological basis of pulmonary gas exchange: implications for clinical interpretation of arterial blood gases. Eur Respir J 2015; 45: 227-243. No. 9: Robertson H.T. Dead space: the physiology of wasted ventilation. Eur Respir J 2015; 45: 1704-1716.
}

Received: May 122015 | Accepted after revision: July 082015 | First published online: Sept 042015

Conflict of interest: None declared.

Copyright OERS 2015 


\section{Introduction}

In pulmonary hypertension $(\mathrm{PH})$, the increased pulmonary arterial load is a key determinant of right ventricular (RV) dysfunction and failure, which in turn is strongly related to patient survival $[1,2]$. Although a complete description of the arterial load can be accomplished by evaluation of the input impedance in the frequency domain $[3,4]$, this approach is complex and more simple time domain measures of the arterial load are often used, particularly in clinical practice. There are two major haemodynamic components of pulmonary arterial load, a steady component and a pulsatile component, which depend upon different parts and properties of the pulmonary arterial tree [5, 6]. The steady component of load can be represented simplistically by mean pulmonary artery pressure (mPAP), which is influenced by the calibre of small distal resistive arteries as reflected in the pulmonary vascular resistance (PVR). The pulsatile component of load is influenced by the elastic properties of the pulmonary arterial tree, which can be estimated by the total arterial compliance $(C)[6,7]$. The product $P V R \times C$ characterises the decay of pulmonary arterial pressure in diastole and represents the time-constant of the pulmonary circulation (RC-time, with units of seconds).

Although pulmonary arterial hypertension (PAH) is classically viewed as a disease of the distal resistive vessels, the rise in RV afterload results from an increase in PVR as well as a reduction in C. Thus, accurate assessment of both the steady and pulsatile components of the RV afterload is of critical importance. It has been proposed that a unique feature of the pulmonary circulation is that PVR and total arterial compliance are tightly coupled through an inverse hyperbolic relationship, resulting in a constant RC-time product that prevails in both health and disease [8-21]. A constant RC-time product has important implications for the assessment of RV afterload, namely that PVR and total arterial compliance are redundant measurements and knowledge of one enables the derivation of the other. Exceptions to the constant RC-time product have recently been documented, for example in PH-related to left heart disease [22-25], prompting a re-evaluation of the concept of RC-time constancy [26-29].

The aim of this review is to describe how the steady and pulsatile components of the RV load are quantified, how PVR and total arterial compliance are related in the pulmonary circulation, and the methodological limitations of these measurements in clinical practice.

\section{Arterial components of right ventricular load \\ Overview}

The right ventricle ejects blood intermittently into the pulmonary circulation, and this explains why pulmonary arterial pressure is pulsatile. In a hydraulic system where the flow is pulsatile, the relationship between pulsatile flow and pulsatile pressure includes not only the resistance to flow afforded by friction but also the opposition to flow due to both vascular elasticity and the inertia of blood mass [3-7, 30]. Thus, the pulmonary arterial load is influenced by distributed compliant and inertial properties. The pulmonary tree is a system of branching tubes with wave travel and reflection (a transmission line network). The characteristic impedance governs the pressure-flow relationship in the proximal pulmonary arterial until the arrival of the first pressure wave reflection, and is a function of total arterial compliance and blood inertance. Although inertance has a more important role at the pulmonary artery level than at the aortic level, clinical studies most often neglect its contribution to load [7, 31]. It is estimated that the pulsatile component of the pulmonary arterial load accounts for $\sim 25 \%$ of RV power on average, as compared with $\sim 10 \%$ in a systemic arteries-left ventricle coupled system $[10,11]$.

As summed up by REUBEN [32]: "Although the concept of the pulmonary arterial system as a lumped system is an oversimplification of reality, it is believed to be valid to a first approximation (...)". Indeed, in the vast majority of the clinical studies performed in the field, a simplified model of arterial load based on the two-element Windkessel model is used. In this lumped haemodynamic model, the arterial system is described in terms of two elements: PVR and C. Although the two-element Windkessel model is clearly an oversimplification of reality, it remains invaluable from the clinical viewpoint and is regarded as providing an adequate description of the arterial system, at least during diastole. However, it must be kept in mind that the model has important limitations: mainly that pulse wave velocity, characteristic impedance and wave reflection are not included in this model [7-9, 33]. For these reasons, the two-element Windkessel model does not apply well at high frequency and during the systolic period. In an attempt to improve the description of pressure and flow in systole, the more complex three-element Windkessel model has been used, which takes into account characteristic impedance [7].

As compared with the normal systemic circulation, the normal pulmonary circulation is characterised by its low resistance and high compliance vasculature with great capabilities for distension and recruitment in reserve. Various physiological mechanisms help preserve low pulmonary arterial load and thus protect both RV function and capillary pressure. Increased arterial load has deleterious consequences on RV 
function and gas exchange. Before discussing the way RC-time is obtained, it is necessary to recall the pathophysiological basis of PVR and total arterial compliance in health and PH states.

\section{Pulmonary vascular resistance}

Physicians are familiar with the concept of PVR, which is a measure of the extent to which the pulmonary circulation "resists" cardiac output (CO). This resistance to mean flow is a function of the blood viscosity and the size and number of vessels [31]. Poiseuille's law predicts that PVR increases in proportion to blood viscosity and inversely with luminal radius to the fourth power. The mean driving pressure of mean pulmonary flow is the transpulmonary pressure gradient, namely the mPAP minus the mean downstream zero-flow pressure (Pzf), which is usually assumed to be equal to pulmonary arterial wedge pressure (PAWP) or left atrial pressure. The driving pressure is related to the frictional resistance of the blood when flow is induced. The standard formula for calculating PVR (based on Ohm's law of electrical circuits) is as follows.

$$
\mathrm{PVR}=\frac{(\mathrm{mPAP}-\mathrm{PAWP})}{\mathrm{CO}}
$$

In precapillary $\mathrm{PH}$, increased PVR occurs as consequence of proliferative remodelling and vasoconstriction of the pulmonary artery in response to chronic pulmonary vascular injury [34]. In pure post-capillary $\mathrm{PH}$, increased mPAP occurs as consequence of increased PAWP while PVR is normal.

The standard PVR equation is integral for the clinical evaluation of pulmonary vascular disease and is a component of the current diagnostic criteria for precapillary $\mathrm{PH}$, which is defined by $\mathrm{mPAP} \geqslant 25 \mathrm{mmHg}$, PAWP $\leqslant 15 \mathrm{mmHg}$ and PVR $>3$ Wood units [35]. Whether PAWP represents the true Pzf is fundamental to the physiological validity of the PVR equation. It is not easy to measure Pzf in humans. In health, PAWP appears to be a reasonable estimate of Pzf, which is further supported by the very small gradient between diastolic pulmonary artery pressure (dPAP) and PAWP, since Pzf must be confined between $\mathrm{dPAP}$ and PAWP. The same reasoning holds true for venous post-capillary PH where the dPAP-PAWP gradient is small $[35,36]$. However, hypoxia and pulmonary vascular diseases leading to precapillary $\mathrm{PH}$ may alter the validity of this assumption, with Pzf being significantly above PAWP, as demonstrated when studying either the pressure intercept of the mPAP-CO linear plots when flow is increased or the pulmonary artery pressure (PAP) decay curve after balloon occlusion [37-42]. In cases where Pzf is significantly higher than PAWP, the true PVR will be overestimated if one uses equation 1 [31]. Finally, it must be noted that some studies have focused on total pulmonary resistance, where a zero PAWP is assumed, which also leads to major overestimation of the true PVR.

If one is interested in quantifying the severity of pulmonary vascular disease per se, as reflected in the geometric reduction in cross-sectional luminal area (i.e. hindrance), adjustment for haematocrit (which is the main determinant of blood viscosity) might be desirable particularly when extremes of haematocrit levels are encountered [37]. Other conceptual limitations of the standard PVR equation include assumptions that blood behaves as a Newtonian fluid and that the arteries behave as rigid tubes while they are both collapsible and distensible. Because normal pulmonary vessels are highly distensible, a more complex curvilinear fit of the mPAP versus CO relationship may be used [43], but it must be noted that the multipoint pressure-flow relationship remains linear over physiological ranges of flow under most physiological, pharmacological and pathological conditions [31, 37].

\section{Compliance}

During systole, a fraction of the ejected stroke volume (SV) is stored in the compliant pulmonary arteries, which is equivalent to charging a capacitor in electronics. During diastole, the blood stored in the compliant pulmonary arteries is released. The systolic storage of this reservoir volume dampens pulsatile RV ejection and decreases both RV stroke work (and thus energy expenditure) and mean RV ejection pressure [7, 44, 45]. Total arterial compliance $(\mathrm{C})$ can be viewed as the storage capacity of the entire pulmonary arterial tree and is defined as the increase in blood volume $(\Delta \mathrm{V})$ in the arterial system that produces a unit increase in arterial transmural pressure $(\Delta \mathrm{P})$ :

$$
\mathrm{C}=\frac{\Delta \mathrm{V}}{\Delta \mathrm{P}}
$$

It can be appreciated that total arterial compliance is difficult to measure in vivo based on the above formula. Although $\Delta \mathrm{P}$ can be readily approximated using the pulmonary arterial pulse pressure (PApp), direct measurement of $\Delta \mathrm{V}$ is not possible since a fraction of the stroke volume (SV) is discharged out of 
the arterial system during RV ejection. Despite this obvious limitation, total arterial compliance is often estimated empirically in clinical practice as:

$$
\text { Pulmonary arterial capacitance }=\frac{\mathrm{SV}}{\mathrm{PApp}}
$$

Given that SV exceeds the reservoir volume of the compliant pulmonary arteries, previous studies have documented that the SV/PApp ratio may significantly overestimate total arterial compliance [46, 47]. Indeed, SEGERS et al. [46] first showed that SV/PApp overestimates total arterial compliance by $81 \%$ in pigs and $60 \%$ in dogs when total arterial compliance was calculated using the reference method, namely the pulse pressure method. Similarly, Muthurangu et al. [47] also reported that SV/PApp overestimates total arterial compliance by $61 \%$ in patients with suspected $\mathrm{PH}$ or congenital heart disease. As a result, authors initially warned against the use of the SV/PApp ratio as an estimate of total arterial compliance [7, 33]. Finally, total arterial compliance must be distinguished from local arterial compliance, which can be estimated by modalities such as magnetic resonance imaging and intravascular ultrasound [48].

Arterial compliance is determined by the prevailing MPAP and by the elasticity of the pulmonary arterial wall, which is mainly determined by the content of elastin (elastic fibres) and collagen (stiffer fibres) in the wall. Arterial compliance decreases when mPAP increases, and this pressure-dependence of pulmonary arterial wall stiffness $(1 / C)$ is due to the nonlinear stress-strain relationship $[48,49]$. In other words, a decrease in total arterial compliance can result from an increase in mPAP without a true change in the elastic properties of the pulmonary arterial wall. Arterial compliance can also decrease when the pulmonary arterial wall stiffens due to a decreased elastin/collagen ratio as a result of vascular remodelling $[48,49]$.

\section{Resistance-compliance relationship of the pulmonary circulation}

A common finding among recent studies is an inverse relationship between PVR and total arterial compliance in both health and disease states including $\mathrm{PAH}$, chronic thromboembolic $\mathrm{PH}$ and left heart dysfunction [8-29]. This confirms previous findings from the landmark studies in the field [3, 4, 32, 50-55]. The inverse relationship between PVR and total arterial compliance is usually described by a hyperbolic fit, meaning that a rise in PVR is followed by an inverse reduction in total arterial compliance, and vice versa. This relationship has now been reported by numerous groups, and is preserved during the course of therapy with PAH-specific agents. Because of this hyperbolic relationship between PVR and total arterial compliance, authors have advocated that the product of PVR and total arterial compliance, which has units of time (referred to as RC-time), remains constant in the pulmonary circulation across the entire range of PAP [8-11]. The RC-time represents the time-constant of the pulmonary circulation, which characterises the decay of pulmonary arterial pressure in diastole. A constant RC-time has important implications for quantification of RV afterload. If RC-time is indeed constant, it suggests that PVR and total arterial compliance are essentially redundant measures and knowledge of one is sufficient to derive the value of the other (see later).

A number of explanations have been put forward for the inverse relationship between PVR and total arterial compliance. It is possible that the prevailing mPAP is itself the main determinant of total arterial compliance. In other words, an increase in PVR will lead to an increase in pressure within the vasculature, which in turn leads to a decrease in compliance due to the nonlinear pressure-diameter relationship of the pulmonary arteries [56]. Alternatively, other groups have hypothesised that the inverse relationship may be explained by the longitudinal distribution of resistance and compliance vessels of the pulmonary circulation $[10,11]$. In the pulmonary circulation, the proximal pulmonary arteries account for proportionally less of the total arterial compliance compared with the aorta in the systemic circulation. Thus, it has been suggested that distal pulmonary arteries have not only a resistive function but also a significant capacitive function and contribute to overall compliance.

An often neglected issue is that the accurate interpretation of the precise functional relationship between two calculated variables such as PVR and SV/PApp requires caution. Vascular resistance is a haemodynamic variable that is frequently mathematically coupled, probably because it is a derived variable [57-60]. Furthermore, there may be a strong mathematical coupling related to the fact that PVR and SV/PApp share SV in the numerator of one and the denominator of the other. In other words, the SV versus $1 / \mathrm{SV}$ relationship is by definition hyperbolic and the relationship between resistance and total arterial compliance is prone to mathematical coupling because SV appears in both the dependent and independent variable. An additional source of mathematical coupling is that mPAP and PApp have an almost 1:1 ratio. 


\section{Prognostic value of PVR and SV/PApp in PH}

Both PVR and SV/PApp have been demonstrated to be of prognostic value in $\mathrm{PH}$, although the data supporting PVR is conflicting. The original National Institutes of Health registry, conducted between 1985 and 1988, and the modern era French registry did not find PVR to be an independent predictor of prognosis in PAH $[60,61]$. However, the large American REVEAL registry showed that extremely high PVR $>32$ Wood units was independently associated with worse survival in PAH [62]. PVR may not be a consistent marker of prognosis in PAH since the main determinant of outcomes is RV function, or the ability of the RV to adapt to the increase in afterload. Accordingly, haemodynamic markers of RV function such as $\mathrm{CO}$ and right atrial pressure have been consistently demonstrated to be prognostic in PAH $[1,2]$.

SV/PApp ratio is a strong independent predictor of mortality in patients with PAH [63, 64] and in scleroderma-related PAH [65]. In congestive heart failure patients, SV/PApp provides stronger prediction than PVR (univariate analysis), and SV/PApp remains an independent predictor with multivariate analysis while PVR does not [23]. It has been suggested that SV/PApp is an integrated measure of RV afterload that may be superior to PVR in explaining RV failure and in predicting adverse outcomes in the setting of left-sided heart failure $[23,66]$. SV/Papp can also be viewed as lumped parameter that takes into account the contributions of wave reflections and pulsatile load because of the inclusion of PApp in its denominator. When wave reflections return from sites of impedance mismatch to the RV, the RV ejection systolic pressure must increase to exceed this added load resulting in an increase in PApp [6, 26].

\section{RC-time: definition, calculation and reference values} Physiological meaning of RC-time and the Windkessel model

RC-time is a time constant that describes the diastolic decay of the pulmonary arterial pressure curve. The concept of RC-time comes from the Windkessel model where the decrease in diastolic pressure is governed by PVR and total arterial compliance. In 1827, Weber first baptised the Windkessel elastic function of aorta. A Windkessel was an air (top) and water (bottom) filled reservoir, similar to that behind the pump of an old-fashioned fire hose, the purpose of which was to transform the rhythmic water output of the plunger strokes into an almost continuous waterjet $[7,44]$. It was Frank who popularised the model [45] and viewed the arterial system as a lumped, two-element Windkessel, with resistance and compliance elements disposed in parallel.

After closure of the pulmonary valve, there is no inflow into the pulmonary arterial system. When pressure and flow are in steady-state, the model assumes that the diastolic pulmonary arterial pressure decay is well fitted by a single exponential function of time, $\mathrm{P}(\mathrm{t})$, with the time constant (RC, expressed in units of time), according to the following equation [59]:

$$
\mathrm{P}(\mathrm{t})=\left(\mathrm{P}_{0}-\mathrm{P}_{\text {asymptote }}\right) \mathrm{e}^{-\mathrm{t} / \mathrm{RC}}+\mathrm{P}_{\text {asymptote }}
$$

$\mathrm{P}_{0}$ is the pressure at the onset of analysis ( $t=0$, corresponding to the time at or immediately after the pulmonary arterial dicrotic notch pressure) and $\mathrm{P}_{\text {asymptote }}$ is the theoretical pressure at the infinite time when the exponential decline is completed [59]. Thus, the RC-time represents the time necessary for pressure to decrease in value to $36.8 \%(1 / \mathrm{e})$ of the $\mathrm{P}_{0}-\mathrm{P}_{\text {asymptote }}$ difference. It will take theoretically three RC-times to complete $95 \%$ of this pressure change (fig. 1).

\section{Calculation of RC-time}

The RC-time product is most commonly calculated using an empiric formula where equation 1 is used to calculate PVR and where total arterial compliance is estimated from SV/PApp (equation 3). Doing this, the basic principles underlying the Windkessel function, namely the exponential decay of pulmonary arterial pressure in diastole (equation 4) is neglected, and PVR and total arterial compliance values are fixed a priori. It is assumed that both PVR (equation 1) and SV/PApp are reliable estimates of true PVR and total arterial compliance, respectively, and that their product accurately reflects the RC-time constant of the Windkessel model. From this empiric method, several groups have documented RC-time values [8-29]. The main studies, corresponding study populations and empirical estimates of RC-time are detailed in table 1.

Alternative methods of calculating RC-time have been described. To the best of our knowledge, the pulmonary arterial RC-time was first calculated in 1959 by ENGELBERG and DuBois [50] using the semi-logarithmic method in rabbit lungs. In 1971, REUBEN [32] used the same method to calculate RC-time in humans with various forms of cardiac and pulmonary diseases. The semi-logarithmic method consists of 


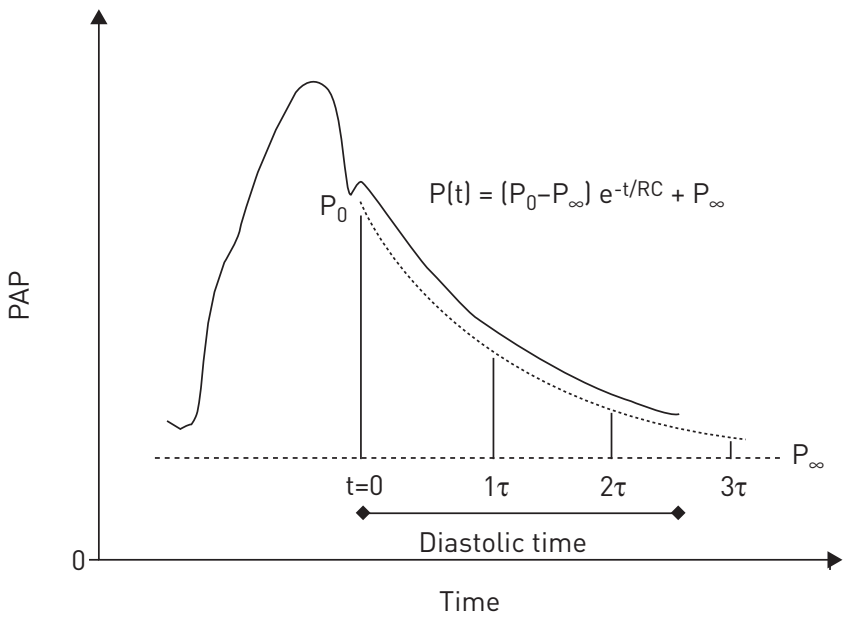

FIGURE 1 A schematic representation that assumes monoexponential pulmonary artery pressure (PAP) decline in diastole from pulmonary arterial notch pressure to end-diastolic pulmonary arterial pressure in pulmonary hypertension. A meaningful Windkessel function implies a zero-flow pressure asymptote at infinite time $\left(P_{\infty}\right)$ slightly below diastolic PAP, with $95 \%$ of the pulmonary arterial notch pressure $\left(P_{0}\right)$ minus $P_{\infty}$ difference achieved at end-diastole, i.e. a time constant of the Windkessel $(\tau)$ circa one third of diastolic time.

a log-transformation of equation 4 and the RC-time is calculated as follows:

$$
\mathrm{RC}=\frac{\text { Diastolic time }}{\ln \left(\mathrm{P}_{0}-\mathrm{P}_{\text {asymptote }} /\left(\mathrm{dPAP}-\mathrm{P}_{\text {asymptote }}\right)\right)}
$$

The PAWP or the left ventricular (LV) filling pressure (when available) is used as $\mathrm{P}_{\text {asymptote, and this also }}$ allows calculation of PVR. Then total arterial compliance is calculated as the RC-time/PVR ratio. The main studies, corresponding study populations and semi-logarithmic estimates of RC-time are detailed in table 2.

\section{Conflicting results on $\mathrm{RC}$-time value in health and diseases}

Recent studies have proposed the notion of constant RC-time (empiric estimate) of $\sim 0.5-0.7 \mathrm{~s}$ in both health and disease [8-11]. Subsequently, it has emerged that various conditions are associated with significant alterations of RC-time. Recently, TEDFORD et al. [22] documented shortened empiric RC-times in post-capillary $\mathrm{PH}$, thus suggesting an increased pulsatile afterload when compared to precapillary $\mathrm{PH}$ at a given level of PVR. This has been subsequently confirmed by other authors [23-25]. These studies still documented an inverse hyperbolic relationship between PVR and SV/Papp, and supported the concept that the mean $\mathrm{PVR} \times \mathrm{C}$ product was in the $0.25-0.53 \mathrm{~s}$ range in post-capillary $\mathrm{PH}$ [22-25]. Very recently, TEDFORD [26] has further challenged the paradigm of constant RC-times in normotensive subjects versus $\mathrm{PAH}$ patients. Indeed, a shortened pulmonary RC-time was documented in 415 subjects with mPAP $\leqslant 25 \mathrm{mmHg}$ (mean RC-time=0.36 s) as compared with 593 subjects with PAH (RC-time=0.53 s) [26].

Using the semi-logarithmic method (table 2), the recovered RC-time averaged 0.16-0.39s in normal subjects but was higher in those with $\mathrm{PH}$, averaging $0.21-0.83 \mathrm{~s}$ (table 2) [32, 51-55]. The shorter RC-times derived from the semi-logarithmic method would be more consistent with more complete expression of the Windkessel function compared with RC-times calculated from empiric estimates. In some studies performed in patients with $\mathrm{PH}$, the RC-times calculated by the semi-logarithmic method are actually similarly as long as RC-times obtained with the empirical method.

\section{RC-time: a critical evaluation and new proposal} Is the RC-time truly constant?

While it is clear that RC-times can no longer be considered constant when precapillary $\mathrm{PH}$, post-capillary $\mathrm{PH}$ and controls are compared, constancy of RC-time has been suggested within a given disease subgroup. Unfortunately, a critical analysis of the data may also challenge this notion. Constancy implies a narrow range of RC-time values. This is not supported by most of the data, which show a large SD and thus a large coefficient of variation $(100 \times \mathrm{SD} /$ mean). In numerous studies (table 1$)$, the RC-time coefficient of variation is $>30 \%$, which reflects great variation of RC-time within the sample. One may simply consider the meaning of a $30 \%$ coefficient of variation of say resting heart rate in a given population with a mean 
TABLE 1 The RC-time in normotensive subjects and in patients with PH estimated from PVR and SV/PApp

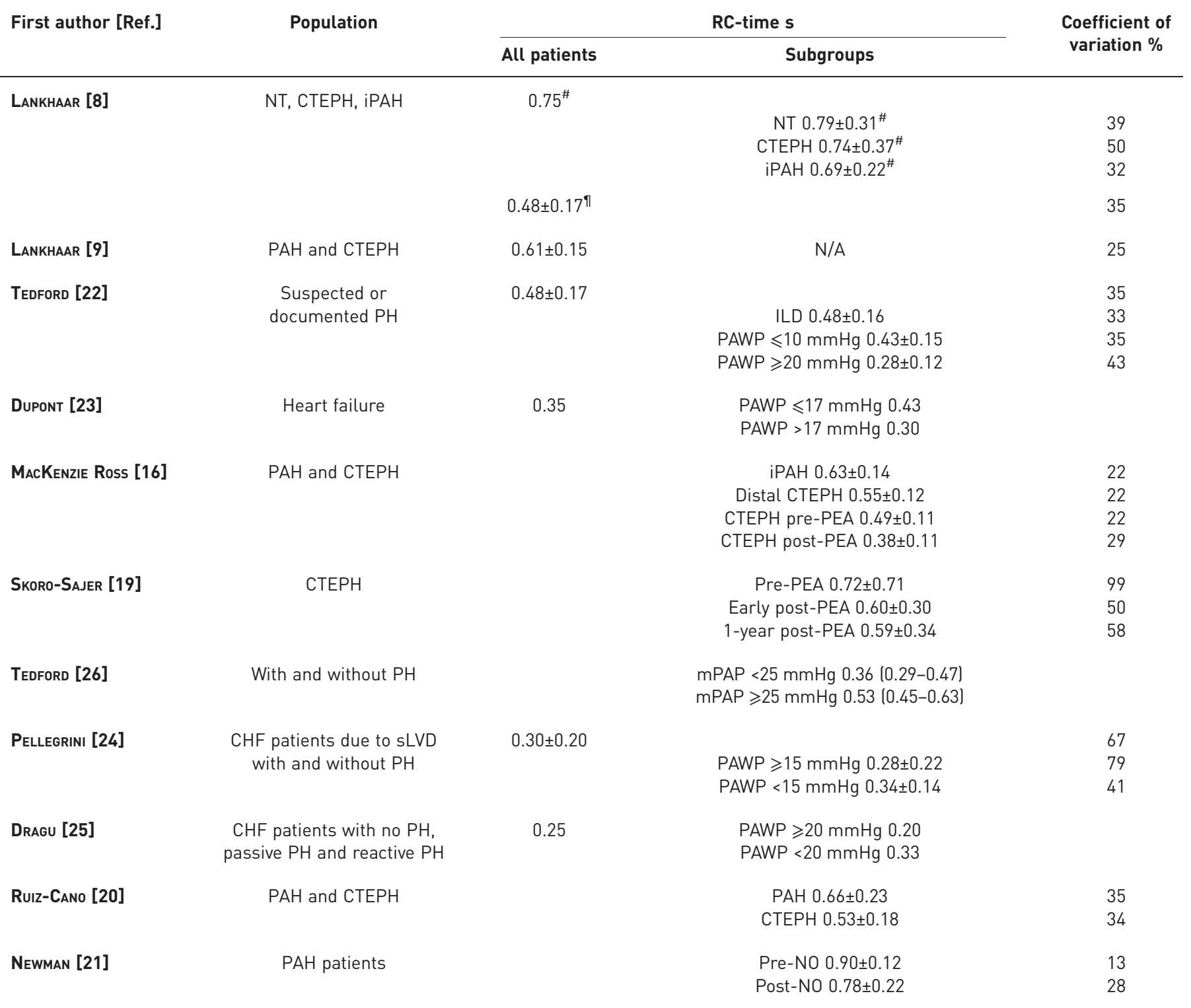

Data are presented as mean, mean \pm SD or mean (range). RC-time: time constant of the monoexponential pulmonary artery pressure decay; $\mathrm{PH}$ : pulmonary hypertension (mean pulmonary artery pressure (mPAP) $\geqslant 25 \mathrm{mmHg}$ ); PVR: pulmonary vascular resistance calculated with zero-flow pressure $(\mathrm{Pzf})=$ pulmonary artery occlusion/wedge pressure (except [8] where $\mathrm{Pzf}=0$ ); SV/Papp: stroke volume/pulmonary arterial pulse pressure; NT: normotensive subjects with mPAP $<25 \mathrm{mmHg}$; CTEPH: chronic thromboembolic pulmonary hypertension; iPAH: idiopathic pulmonary arterial hypertension; N/A: not applicable; ILD: interstitial lung disease; PAWP: pulmonary arterial wedge pressure; PEA: pulmonary thromboendarterectomy; mPAP: mean pulmonary artery pressure; CHF: congestive heart failure; sLVD: systolic left ventricular dysfunction; PAH: pulmonary arterial hypertension; NO: nitric oxide; The total arterial compliance was empirically estimated by using the SV/PApp lexcept

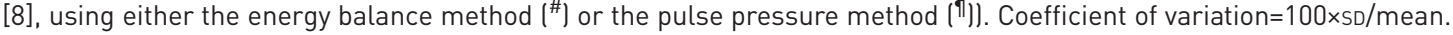

of 80 beats. $\mathrm{min}^{-1}$, assuming a normal distribution. The $95 \%$ confidence interval of $33-127$ beats $\mathrm{min}^{-1}$ certainly points to major variation of resting heart rate in the sample rather than "constancy", with the upper limit of the $95 \%$ confidence interval being four-times greater than the lower limit. Our group has recently documented major scattering of RC-time in the precapillary $\mathrm{PH}$ subgroup (fig. 2) [27]. In post-capillary $\mathrm{PH}, \mathrm{RC}$-time decreases linearly with increasing left atrial pressure [22-24]. Finally, in precapillary $\mathrm{PH}$, it has been suggested that at high values of $\mathrm{PVR}$, compliance reaches a minimum and that further increases in PVR would result in an increase in RC-time [17]. The above observations have led to concerns and cast doubt regarding the RC-time constancy paradigm even among specific disease subgroups. 
TABLE 2 The RC-time in normotensive (NT) subjects and in patients with pulmonary hypertension $(\mathrm{PH})$ estimated using the semi-logarithmic method

\begin{tabular}{|c|c|c|c|c|}
\hline \multirow[t]{2}{*}{ First author [Ref.] } & \multicolumn{2}{|c|}{ RC-time s } & \multicolumn{2}{|c|}{$\mathrm{C}, \mathrm{mL} \cdot \mathrm{mmHg}^{-1}$} \\
\hline & NT & $\mathrm{PH}$ & NT & $\mathrm{PH}$ \\
\hline Reuben [32] & $0.208(0.092-1.090)$ & $0.383(0.174-0.690)$ & 2.87 & 0.85 \\
\hline KIRBY [51] & $0.39 \pm 0.23$ & $0.83 \pm 0.42$ & $5.66 \pm 1.58$ & $3.08 \pm 1.96$ \\
\hline Senzaki [52] & $0.23 \pm 0.14$ & $0.56 \pm 0.04$ & N/A & N/A \\
\hline BASNEt [53] & $0.16 \pm 0.06$ & $0.21 \pm 0.11$ & $1.53 \pm 0.17$ & $0.95 \pm 0.06$ \\
\hline
\end{tabular}

Data are presented as mean, mean \pm SD or mean (range). The values were obtained from individual patient data using equation 5 and are presented as published in the original articles. RC-time: time constant of the monoexponential pulmonary artery pressure decay; $\mathrm{C}$ : the estimated total arterial compliance calculated as $\mathrm{C}=\mathrm{RC}$-time/pulmonary vascular resistance; N/A: not applicable.

Despite the irrefutable inverse relationship between PVR and SV/PApp, we suggest that there remains significant scatter around the hyperbolic curve fits and RC-time [26]. The accurate measurement of PApp using fluid-filled catheters is especially challenging and a lot of this scatter may also be due to measurement error [26].

Are estimates of RC-time too long?

As previously discussed, RC-time values in the range of $0.50-0.70 \mathrm{~s}$ have been widely documented in numerous recent studies performed in precapillary PH (table 1). Some studies using the semi-logarithmic method have also documented a similarly long RC-time in PH (table 2). Assuming a heart period of $0.80 \mathrm{~s}$ (RV ejection time $\sim 0.30 \mathrm{~s}$, diastolic time $\sim 0.50 \mathrm{~s}$ ) and a RC-time of $0.60 \mathrm{~s}$ this implies: 1 ) that $<63.2 \%$ (i.e. $100-36.8 \%)$ of the Windkessel function has been manifested at end-diastole; and 2) that completion of the Windkessel function may theoretically only be observed after $1.8 \mathrm{~s}$. This supports incomplete expression of the Windkessel function in $\mathrm{PH}$, with RV ejection occurring at a time when pulmonary arterial emptying is not completed. Because the accurate measurement of PApp using fluid-filled catheters is especially challenging, one possibility is that RC-time may have been overestimated due to measurement error [26]. This is unlikely to be the main explanation as short RC-time has been documented in non-PH subjects and in post-capillary PH. The non-normal distribution of PVR and SV/PApp could also contribute to an abnormally high and unreliable value of the product of their mean values [27]. Initial studies performed in precapillary $\mathrm{PH}$ have used total pulmonary resistance $(\mathrm{mPAP} / \mathrm{CO})$ instead of PVR and this may have also contributed to overestimation of true PVR and thus, to abnormally long RC-time reference values.

In keeping with the hypothesis of a useful Windkessel function in $\mathrm{PH}$, and in keeping with the widely admitted notion that pulmonary flow is near-zero at end-diastolic pressure $[67,68]$, here we suggest that the RC-time may have been overestimated, mainly due to the marked overestimation of resistance by using PVR, and, to a lesser extent, due to overestimation of total arterial compliance using SV/PApp. Indeed, if

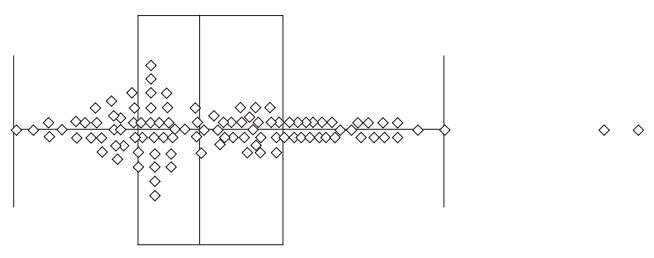

\begin{tabular}{|c|c|c|c|c|}
\hline 200 & 400 & 600 & 800 & 1000 \\
\hline & & & & \\
\hline
\end{tabular}

FIGURE 2 Box-and-whisker plots showing extreme scatter around the median (middle line) of pulmonary arterial time constant (RC) in patients with precapillary pulmonary hypertension ( $n=104)$. The time constant RC was calculated by multiplying the pulmonary vascular resistance by stroke volume over pulmonary arterial pulse pressure ratio. The central box represents the values from the lower to upper quartiles (25th-75th percentile). The horizontal line extends from the minimum to the maximum value, excluding outside values. An outside value is defined as a value that is smaller than the lower quartile minus 1.5 times the interquartile range, or larger than the upper quartile plus 1.5 times the interquartile range (inner fences). Information from [27]. 
Pzf pressure is higher than PAWP in the setting of pulmonary vascular disease, especially precapillary $\mathrm{PH}$, the true PVR will be overestimated if one uses equation 1.

In the setting of PAH, increases in resting dPAP are responsible for increased mPAP [67, 68], and are related to pulmonary rarefaction, vasoconstriction, vascular remodelling and intravascular plexiform or thrombotic lesions at the level of small pulmonary arteries [34]. The loss of pulmonary vessels, the major narrowing of open vessels, and the heightened sensitivity to hypoxia and other vasoconstrictive agents [34] may well underlie an increase in the critical closing pressure at which the flow dramatically decreases and stops (i.e. Pzf). Due to the fact that pulmonary vessels are collapsible [69], the high Pzf exceeds PAWP and Pzf becomes the effective outflow pressure.

Similarly, SV/PApp may also overestimate total arterial compliance when SV exceeds the Windkessel reservoir volume [44, 46, 47]. Finally, some studies have used total pulmonary resistance as an estimation of PVR, implying that Pzf is $0 \mathrm{mmHg}$. Clearly, Pzf cannot be lower than PAWP (or left atrial pressure) and we concur with other authors $[26,28,70]$ who have advised against the use of a $0 \mathrm{mmHg}$ Pzf, which leads to unrealistically high RC-time values.

\section{Improving the estimation of RC-time}

Because pulmonary flow is near-zero at end-diastole $[67,68]$, near complete expression of the Windkessel function is thought to occur during diastole, and it is anticipated that the order of magnitude of RC-time should be much shorter than diastolic time (perhaps approximately one third of diastolic time since three RC-times correspond to $95 \%$ of the pressure fall to asymptotic pressure) [71]. In this setting, RC-time will be constrained by diastolic time if near complete expression of the Windkessel function occurs at varying heart rates. At the systemic circulation level, a meta-analysis involving 36 major studies on Windkessel parameters has documented that the diastolic time/systemic RC-time ratio was independent of body mass in mammals, and this may be viewed as a contributory factor in the left ventricular-arterial coupling [72]. In the right-sided counterpart, it seems logical that diastolic time (and thus heart rate) and the mechanical properties of the pulmonary arterial tree (PVR and total arterial compliance) may also be mutually constrained. The right ventricle and pulmonary arterial tree form a strongly coupled unit. Recently, an unusually strong linear relationship between heart rate and MPAP has been described in $\mathrm{PH}$ patients performing exercise [73]. In this respect, a fixed diastolic time/RC-time ratio may be especially important on exercise, as delayed Windkessel function may compromise RV ejection if pulmonary arteries emptying is not completed.

As recalled by BASNET et al. [53]: "The time constant can be calculated from the pressure curve between any two points during diastole". Indeed, the fundamental property of a monoexponential function is that, at each time, the rate of change of a given variable (here pressure) is proportional to the value of the variable itself at this time. In other words, in cases where decay of diastolic pressure is truly a monoexponential function of time, there must be a significant negative linear relationship between the instantaneous rate of pressure decline and pressure itself. The slope of the relationship $(-1 / \mathrm{Tau})$ can be calculated using the so-called derivative method popularised by RAFF and GLANTZ [74] to help calculate the LV relaxation time constant. Overall, numerous, mathematical tools that take the pressure asymptote into account are available to reliably calculate the time constant (Tau) of exponential decay processes [59, 74, 75]. Cardiac physiologists are well aware of such tools allowing the accurate calculation the LV relaxation time constant, as has been documented in many previously published studies. To our knowledge, these mathematical tools remain to be used to study pulmonary arterial RC-time. These mathematical tools will also allow the validity of the exponential model applied to the PAP decay in diastole to be checked [76], and thus the validity of the RC-time model in both health and disease. Because pulmonary capillary pressure/flow remain pulsatile in the normal pulmonary circulation, the classic Windkessel concept of fully dampening pulsatility and providing steady pressure/flow at the capillary level may not be fully applicable to healthy subjects.

\section{Conclusion}

In $\mathrm{PH}$, the increased pulmonary arterial load is a key determinant of RV dysfunction and thus prognosis. PVR and total arterial compliance modulate the steady and pulsatile component of pulmonary arterial load, respectively. In the pulmonary circulation, PVR is inversely related to total arterial compliance, such that an increase or decrease in PVR is followed by a respective decrease or increase in total arterial compliance. Assuming that there is a hyperbolic relationship between PVR and total arterial compliance, recent studies have popularised the concept that the RC-time of the pulmonary circulation is "constant" in health and diseases. However, emerging evidence suggests that this concept should be challenged, and different pathophysiological states are clearly associated with significant alteration in RC-times, such as the shortened RC-times documented in post-capillary $\mathrm{PH}$ and in normotensive subjects. Furthermore, reported RC-times in the literature have consistently demonstrated significant scatter around the mean. 
Another potential problem with pulmonary RC-time is the methodology that is employed to calculate it. Empiric estimates of PVR and total arterial compliance may be prone to errors. In precapillary $\mathrm{PH}$, the true PVR can be overestimated if one uses the standard PVR equation because Pzf may be significantly higher than PAWP. Furthermore, SV/PApp may also be associated with a potential overestimation of true total arterial compliance. With these considerations, the recently published empiric RC-times might be overestimates and are too long to be consistent with a useful Windkessel function. The inaccuracies associated with empiric PVR and total arterial compliance estimates may well change opinions on the distribution of compliance and resistance in the lung, and this point deserves further study.

A re-evaluation of the current concept of RC-time is needed, using alternative methodologies that have been employed to study the systemic circulation, and high-fidelity pressure instruments to improve measurement precision. Further studies are needed to clarify some of the inconsistencies of pulmonary RC-time, as this has major implications for our understanding of the arterial load in diseases of the pulmonary circulation.

\section{References}

1 Naeije R, Manes A. The right ventricle in pulmonary arterial hypertension. Eur Respir Rev 2014; 23: 476-487.

2 Vonk-Noordegraaf A, Haddad F, Chin KM, et al. Right heart adaptation to pulmonary arterial hypertension: physiology and pathobiology. J Am Coll Cardiol 2013; 62: Suppl., D22-D33.

3 Milnor WR, Conti CR, Lewis KB, et al. Pulmonary arterial pulse wave velocity and impedance in man. Circ Res 1969; 25: 637-649.

4 Grant BJB, Lieber BB. Clinical significance of pulmonary arterial input impedance. Eur Respir J 1996; 9: 2196-2199.

5 Chemla D, Castelain V, Hervé P, et al. Haemodynamic evaluation of pulmonary hypertension. Eur Respir J 2002; 20: 1314-1331.

6 Castelain V, Hervé P, Lecarpentier Y, et al. Pulmonary artery pulse pressure and wave reflection in chronic pulmonary thromboembolism and primary pulmonary hypertension. J Am Coll Cardiol 2001; 37: 1085-1092.

$7 \quad$ Westerhof N, Lankhaar JW, Westerhof BE. The arterial Windkessel. Med Biol Eng Comput 2009; 47: $131-141$.

8 Lankhaar JW, Westerhof N, Faes TJ, et al. Quantification of right ventricular afterload in patients with and without pulmonary hypertension. Am J Physiol Heart Circ Physiol 2006; 29: H1731-H1737.

9 Lankhaar JW, Westerhof N, Faes TJ, et al. Pulmonary vascular resistance and compliance stay inversely related during treatment of pulmonary hypertension. Eur Heart J 2008; 29: 1688-1695.

10 Saouti N, Westerhof N, Helderman F, et al. RC time constant of single lung equals that of both lungs together: a study in chronic thromboembolic pulmonary hypertension. Am J Physiol Heart Circ Physiol 2009; 297: H2154-H2160.

11 Saouti N, Westerhof N, Postmus PE, et al. The arterial load in pulmonary hypertension. Eur Respir Rev 2010; 19: 197-203.

12 Bonderman D, Martischnig AM, Vonbank K, et al. Right ventricular load at exercise is a cause of persistent exercise limitation in patients with normal resting pulmonary vascular resistance after pulmonary endarterectomy. Chest 2011; 139: 122-127.

13 de Perrot M, McRae K, Shargall Y, et al. Early postoperative pulmonary vascular compliance predicts outcome after pulmonary endarterectomy for chronic thromboembolic pulmonary hypertension. Chest 2011; 140: 34-41.

14 Kind T, Faes TJ, Vonk-Noordegraaf A, et al. Proportional relations between systolic, diastolic and mean pulmonary artery pressure are explained by vascular properties. Cardiovasc Eng Technol 2011; 2: 15-23.

15 Pagnamenta A, Vanderpool R, Brimioulle S, et al. Proximal pulmonary arterial obstruction decreases the time constant of the pulmonary circulation and increases right ventricular afterload. J Appl Physiol (1985) 2013; 114: 1586-1592.

16 MacKenzie Ross RV, Toshner MR, Soon E, et al. Decreased time constant of the pulmonary circulation in chronic thromboembolic pulmonary hypertension. Am J Physiol Heart Circ Physiol 2013; 305: H259-H264.

17 Tedford RJ, Mudd JO, Girgis RE, et al. Right ventricular dysfunction in systemic sclerosis-associated pulmonary arterial hypertension. Circ Heart Fail 2013; 6: 953-963.

18 Grignola JC, Domingo E. Pulmonary endarterectomy in chronic thromboembolic pulmonary hypertension: How can patients be better selected? World J Cardiol 2013; 5: 18-21.

19 Skoro-Sajer N, Marta G, Gerges C, et al. Surgical specimens, haemodynamics and long-term outcomes after pulmonary endarterectomy. Thorax 2014; 69: 116-122.

20 Ruiz-Cano MJ, Grignola JC, Barberá JA, et al. The distribution of the obstruction in the pulmonary arteries modifies pulsatile right ventricular afterload in pulmonary hypertension. Int J Cardiol 2015; 181: 232-234.

21 Newman JH, Brittain EL, Robbins IM, et al. Effect of acute arteriolar vasodilation on capacitance and resistance in pulmonary arterial hypertension. Chest 2015; 147: 1080-1085.

22 Tedford RJ, Hassoun PM, Mathai SC, et al. Pulmonary capillary wedge pressure augments right ventricular pulsatile loading. Circulation 2012; 125: 289-297.

23 Dupont M, Mullens W, Skouri HN, et al. Prognostic role of pulmonary arterial capacitance in advanced heart failure. Circ Heart Fail 2012; 5: 778-785.

24 Pellegrini P, Rossi A, Pasotti M, et al. Prognostic relevance of pulmonary arterial compliance in patients with chronic heart failure. Chest 2014; 145: 1064-1070.

25 Dragu R, Rispler S, Habib M, et al. Pulmonary arterial capacitance in patients with heart failure and reactive pulmonary hypertension. Eur J Heart Fail 2015; 17: 74-80.

26 Tedford RJ. Determinants of right ventricular afterload (2013 Grover Conference series). Pulm Circ 2014; 4 211-219.

27 Chemla D, Creuzé N, Hoette S, et al. Relationship between the pulsatile and steady component of right ventricular aftertload in patients with precapillary pulmonary hypertension. Am J Respir Crit Care Med 2014: 189; A1899. 

I Respir Crit Care Med 2014: 189; A4717.

29 Grignola JC. Is the time constant of the pulmonary circulation truly constant? Eur Respir J 2014; 43: 1539-1541.

30 Chemla D, Plamann K, Nitenberg A. Towards new indices of arterial stiffness using systolic pulse contour analysis: a theoretical point of view. J Cardiovasc Pharmacol 2008; 51: 111-117.

31 Naeije R, Westerhof N. Pulmonary vascular function. In: Yuan JXJ, Garda JGN, West JB, Hales CA, Rich S, Archer SL, Eds. Textbook of Pulmonary Vascular Disease. New York, Springer 2011: 61-72.

32 Reuben SR. Compliance of the human pulmonary arterial system in disease. Circ Res 1971; 29: 40-50.

33 Westerhof N, Stergiopulos N, Noble MIM. Snapshots of Hemodynamics. An Aid for Clinical Research and Graduate Education. Dordrecht, Kluwer Academic Publishers, 2005.

34 McLaughlin VV, Shah SJ, Souza R, et al. Management of pulmonary arterial hypertension. J Am Coll Cardiol 2015; 65: 1976-1997.

35 Hoeper MM, Bogaard HJ, Condliffe R, et al. Definitions and diagnosis of pulmonary hypertension. J Am Coll Cardiol 2013; 62: Suppl., D42-D50.

36 Vachiéry JL, Adir Y, Barberà JA, et al. Pulmonary hypertension due to left heart diseases. J Am Coll Cardiol 2013; 62 (25 Suppl): Suppl., D100-D108.

37 Naeije R, Chesler N. Pulmonary circulation at exercise. Compr Physiol 2012; 2: 711-741.

38 Leeman M, Lejeune P, Closset J, et al. Nature of pulmonary hypertension in canine oleic acid pulmonary edema. J Appl Physiol 1990; 69: 293-298.

39 Kafi SA, Mélot C, Vachiéry JL, et al. Partitioning of pulmonary vascular resistance in primary pulmonary hypertension. J Am Coll Cardiol 1998; 31: 1372-1376.

40 Fesler P, Pagnamenta A, Vachiéry JL, et al. Single arterial occlusion to locate resistance in patients with pulmonary hypertension. Eur Respir J 2003; 21: 31-36.

41 Kim NH, Fesler P, Channick RN, et al. Preoperative partitioning of pulmonary vascular resistance correlates with early outcome after thromboendarterectomy for chronic thromboembolic pulmonary hypertension. Circulation 2004; 109: 18-22.

42 Lau EM, Vanderpool RR, Choudhary P, et al. Dobutamine stress echocardiography for the assessment of pressure-flow relationships of the pulmonary circulation. Chest 2014; 146: 959-966.

43 Reeves JT, Linehan JH, Stenmark KR. Distensibility of the normal human lung circulation during exercise. Am J Physiol Lung Cell Mol Physiol 2005; 288: L419-L425.

44 Belz GG. Elastic properties and Windkessel function of the human aorta. Cardiovasc Drugs Ther 1995; 9: 73-83.

45 Sagawa K, Lie RK, Schaefer J. Translation of Otto Frank's paper "Die Grundform des Arteriellen Pulses" Zeitschrift für Biologie 37: 483-526 (1899). J Mol Cell Cardiol 1990; 22: 253-254.

46 Segers P, Brimioulle S, Stergiopulos N, et al. Pulmonary arterial compliance in dogs and pigs: the three-element windkessel model revisited. Am J Physiol 1999; 277: H725-H731.

47 Muthurangu V, Atkinson D, Sermesant M, et al. Measurement of total pulmonary arterial compliance using invasive pressure monitoring and MR flow quantification during MR-guided cardiac catheterization. Am J Physiol Heart Circ Physiol 2005; 289: H1301-H1306.

48 Wang Z, Chesler NC. Pulmonary vascular wall stiffness: an important contributor to the increased right ventricular afterload with pulmonary hypertension. Pulm Circ 2011; 1: 212-223.

49 Lau EM, Iyer N, Ilsar R, et al. Abnormal pulmonary artery stiffness in pulmonary arterial hypertension: in vivo study with intravascular ultrasound. PLoS One 2012; 7: e33331.

50 Engelberg J, DuBois AB. Mechanics of pulmonary circulation in isolated rabbit lungs. Am J Physiol 1959; 196: 401-414.

51 Kirby BJ. Pulmonary artery compliance in pulmonary heart disease. Prog Resp Res 1975; 9: 254-260.

52 Senzaki H, Kato H, Akagi M, et al. New criteria for the radical repair of congenital heart disease with pulmonary hypertension in order to avoid postoperative residual pulmonary hypertension. Jpn Heart J 1995; 36: 49-59.

53 Basnet NB, Awa S, Hishi T, et al. Pulmonary arterial compliance in children with atrial and ventricular septal defect. Heart Vessels 2000; 15: 61-69.

54 Senzaki H, Kato H, Akagi M, et al. Relationship between the pulmonary artery index and physiological properties of the pulmonary vascular bed. Jap Circ J 1996; 60: 334-340.

55 Montalescot G, Drobinski G, Meurin P, et al. Effects of prostacyclin on the pulmonary vascular tone and cardiac contractility of patients with pulmonary hypertension secondary to end-stage heart failure. Am J Cardiol 1998; 82: 749-755.

56 Sniderman AD, Fitchett DH. Vasodilators and pulmonary arterial hypertension: the paradox of therapeutic success and clinical failure. Int J Cardiol 1988; 20: 173-181.

57 Archie JP Jr. Mathematic coupling of data: a common source of error. Ann Surg 1981; 193: 296-303.

58 Walsh TS, Lee A. Mathematical coupling in medical research: lessons from studies of oxygen kinetics. $\mathrm{Br} J$ Anaesth 1998; 81: 118-120.

59 Liu Z, Brin KP, Yin FC. Estimation of total arterial compliance: an improved method and evaluation of current methods. Am J Physiol 1986; 251: H588-H600.

60 D'Alonzo GE, Barst RJ, Ayres SM, et al. Survival in patients with primary pulmonary hypertension. Results from a national prospective registry. Ann Intern Med 1991; 115: 343-349.

61 Benza RL, Gomberg-Maitland M, Miller DP, et al. The REVEAL Registry risk score calculator in patients newly diagnosed with pulmonary arterial hypertension. Chest 2012; 141: 354-362.

62 Benza RL, Miller DP, Barst RJ, et al. An evaluation of long-term survival from time of diagnosis in pulmonary arterial hypertension from the REVEAL registry. Chest 2012; 142: 448-456.

63 Mahapatra S, Nishimura RA, Sorajja P, et al. Relationship of pulmonary arterial capacitance and mortality in idiopathic pulmonary arterial hypertension. J Am Coll Cardiol 2006; 47: 799-803.

64 Gan CT, Lankhaar JW, Westerhof N, et al. Noninvasively assessed pulmonary artery stiffness predicts mortality in pulmonary arterial hypertension. Chest 2007; 132: 1906-1912.

65 Campo A, Mathai SC, Le Pavec J, et al. Hemodynamic predictors of survival in scleroderma-related pulmonary arterial hypertension. Am J Respir Crit Care Med 2010; 182: 252-260. 
66 Al-Naamani N, Preston IR, Paulus JK, et al. Pulmonary arterial capacitance is an important predictor of mortality in heart failure with a preserved ejection fraction. JACC Heart Fail 2015; 3: 467-474.

67 Harvey RM, Enson Y, Ferrer MI. A reconsideration of the origins of pulmonary hypertension. Chest 1971; 59: 82-94.

68 Enson Y. Pulmonary heart disease: relation of pulmonary hypertension to abnormal lung structure and function. Bull N Y Acad Sci 1977; 53: 551-566.

69 Permutt S, Riley RL. Hemodynamics of collapsible vessels with tone: the vascular waterfall effect. J Appl Physiol 1963; 18: 924-932.

70 Kottenberg-Assenmacher E, Aleksic I, Eckholt M, et al. Critical closing pressure as the arterial downstream pressure with the heart beating and during circulatory arrest. Anesthesiology 2009; 110: 370-379.

71 Chemla D, Nitenberg A. Total arterial compliance estimated from the time constant of aortic pressure decay: the influence of downstream pressure. FASEB J 2010; 24: Suppl., 1039.5.

72 Westerhof N, Elzinga G. Normalized input impedance and arterial decay time over heart period are independent of animal size. Am J Physiol 1991; 261: R126-R133.

73 Chemla D, Castelain V, Hoette S, et al. Strong linear relationship between heart rate and mean pulmonary artery pressure in exercising patients with severe precapillary pulmonary hypertension. Am J Physiol Heart Circ Physiol 2013; 305: H769-H777.

74 Raff GL, Glantz SA. Volume loading slows left ventricular isovolumic relaxation rate. Evidence of load-dependent relaxation in the intact dog heart. Circ Res 1981; 48: 813-824.

75 Martin G, Gimeno JV, Cosin J, et al. Time constant of isovolumic pressure fall: new numerical approaches and significance. Am J Physiol 1984; 247: H283-H294.

76 Xu D, Olivier B, Mukkamala R. Continuous cardiac output and left atrial pressure monitoring by long time interval analysis of the pulmonary artery pressure waveform: proof of concept in dogs. J Appl Physiol 2009; 106: 651-661. 